\title{
Tabularia
}

\section{Le cartulaire de l'abbaye Saint-Pierre de Préaux : présentation du manuscrit}

The cartulary of the abbey of Saint-Pierre de Préaux: an introduction to the manuscript

\section{Dominique Rouet}

\section{(2) OpenEdition}

\section{Journals}

Édition électronique

URL : http://journals.openedition.org/tabularia/2455

DOI : 10.4000/tabularia.2455

ISSN : 1630-7364

Éditeur :

CRAHAM - Centre Michel de Boüard, Presses universitaires de Caen

Référence électronique

Dominique Rouet, «Le cartulaire de l'abbaye Saint-Pierre de Préaux : présentation du manuscrit », Tabularia [En ligne], Sources en ligne, mis en ligne le 22 octobre 2001, consulté le 03 mai 2019. URL: http://journals.openedition.org/tabularia/2455; DOI : 10.4000/tabularia.2455 


\title{
Le cartulaire de l'abbaye Saint-Pierre de Préaux: présentation du manuscrit
}

\section{The cartulary of the abbey of Saint-Pierre de Préaux: an introduction to the manuscript}

\author{
Dominique ROUET \\ Bibliothèque municipale du Havre \\ 17, rue Jules Lecesne \\ 76600 Le Havre \\ Dominique.Rouet@ville-lehavre.fr
}

Résumé :

L'abbaye bénédictine de Saint-Pierre de Préaux était située dans l'ancien diocèse de Lisieux, au sud-est de Pont-Audemer. Elle fut le monastère patrimonial des comtes de Meulan et a laissé un cartulaire copié au XIII siècle. La copie de cet important manuscrit, conservé aux Archives départementales de l'Eure, sous la cote H 711, a été entreprise durant l'année 1227, puis poursuivie jusqu'au XIV ${ }^{e}$ siècle. Ce cartulaire regroupe un ensemble de notices datant des $\mathrm{XI}^{e}$ et $\mathrm{XII}^{\mathrm{e}}$ siècles, transcrites d'après un cartulaire plus ancien, auxquelles ont été ajoutées des chartes plus récentes.

Mots-clés : Saint-Pierre de Préaux, cartulaire, charte, notice.

Abstract:

The benedictine abbey of Saint-Pierre de Préaux which belonged to the former diocese of Lisieux, was located to the south-east of Pont-Audemer. This monastery, where the counts of Meulan were buried, has left an important cartulary, now kept in Archives départementales de l'Eure (H 711). The copy of this important manuscript begun first in 1227 and was continued till the 14th $\mathrm{c}$. It gathers several notices dating from the 11 th and 12 th c., extracted from an earlier cartulary, and other charters from the beginning of the 13th century.

Keywords: Saint-Pierre de Préaux, cartulary, charter, notice.

\section{L'abbaye Saint-Pierre de Préaux}

Aujourd'hui totalement disparue, l'abbaye Saint-Pierre de Préaux se situait en lisière de l'ancien diocèse de Lisieux, à quelques kilomètres au sud-est de PontAudemer ${ }^{1}$. Les origines de ce monastère remontent à l'époque des «saints pères de

1. Pont-Audemer, Eure. 
Fontenelle», avant l'arrivée des Normands, puisqu'on possède une mention de son existence dans le testament d'Anségise abbé de Fontenelle ${ }^{2}$. Préaux se trouve fondée à un endroit stratégique, à proximité d'un fisc de Saint-Germain-des-Prés, la Villa supra mare identifiée à l'actuel Saint-Germain-Village ${ }^{3}$.

Détruite par les Normands puis restaurée en 1034, Préaux bénéficie à nouveau de sa situation, à proximité d'un pont sur la Risle, lieu de passage obligé, où se développe un important marché qui devient au début du XI ${ }^{\mathrm{e}}$ siècle Pont-Audemer. C'est à Onfroi de Vieilles, secondé par l'abbé Gradulfe de Fontenelle, que l'on doit cette restauration: Saint-Pierre de Préaux devient ainsi fille de Fontenelle qui lui donne ses premiers moines et ses trois premiers abbés. Onfroi, à l'origine de la famille des Beaumont-Meulan, fait de Saint-Pierre de Préaux une abbaye familiale qui deviendra la nécropole des comtes de Meulan.

Au XII ${ }^{e}$ siècle, sous le gouvernement de sages et doctes abbés venus, à partir de la seconde moitié du siècle, du Bec, Préaux étend ses dépendances, fruits de la générosité des comtes de Meulan notamment: du Bessin au pays de Bray ${ }^{4}$, de la vallée de la Risle aux coteaux de la Seine dominant Meulan et outre-Manche du Dorset au Norfolk.

Du chartrier, qui eut à subir très tôt de graves dommages, il ne demeure plus aujourd'hui que quelques vestiges conservés aux Archives départementales de l'Eure, mais il subsiste également deux importants cartulaires. Celui qui retient notre attention date du début du XIII ${ }^{\mathrm{e}}$ siècle; un second manuscrit copié au XV $\mathrm{XV}^{\mathrm{e}}$ siècle reprend une partie du cartulaire précédent qu'il enrichit de nombreux actes de la seconde moitié du XVe siècle 5 .

2. Gesta Sanctorum Patrum Fontanellensis coenobii, éd. Fernand LOHIER et Dom Jean LAPORTE, Paris/ Rouen, Société de l'Histoire de Normandie, 1936, chap. VIII, par. 7 ; voir aussi LAPORTE, Jean, «Une variété de rouleaux des morts monastiques. Le testament d’Anségise (833)», Revue Mabillon, 1952, p. 45-55. Sur la fondation de l'abbaye par Onfroi de Vieilles et Gradulfe de Fontenelle, voir l'Inventio et miracula sancti Vulfranni, éd. Dom Jean LAPORTE, Rouen/Paris, Société de l'Histoire de Normandie (Mélanges, vol. 14) 1938, p. 51-52.

3. Voir LOHRMANN, Dietrich, «Le moulin à eau dans le cadre de l'économie rurale de la Neustrie (VIII ${ }^{\text {- }}$ IX siècles) ", in La Neustrie: les pays au nord de la Loire de 650 à 850. Colloque historique international, Sigmaringen, 1989, t. I, p. 380-381.

4. Sur le temporel de Saint-Pierre de Préaux, voir GAZEAU, Véronique, «Le temporel de l'abbaye Saint-Pierre des Préaux au XI ${ }^{e}$ siècle ", in Recueil d'études en hommage à Lucien Musset (Cahier des annales de Normandie, n² 23), Caen, 1990, p. 237-253; MUSSET, Lucien, «Autour de la Basse-Dive, le prieuré de Saint-Pierre de Rouville et ses dépendances d'après ses plus anciennes chartes », Bulletin de la Société des Antiquaires de Normandie, t. 59, 1990, p. 247-258; RouET, Dominique, «Une dépendance de l'abbaye Saint-Pierre de Préaux: le prieuré Sainte-Radegonde de Neufchâtel-enBray d'après les sources de l'abbaye de Préaux ", Annales de Normandie, 1999, nº 5, p. 515-538.

5. Il s'agit respectivement des manuscrits H 711, Arch. dép. Eure, et nouv. acq. lat. 1929 de la Bibliothèque nationale de France. 


\section{Présentation du manuscrit}

Le premier cartulaire ${ }^{6}$ de Saint-Pierre de Préaux, conservé aux Archives départementales de l'Eure, sous la cote H 711, présente les dimensions d'un petit in folio ${ }^{7}$. On recense dans ce volume 640 chartes dont les dates s'échelonnent de 1034 à 1494 ; il est relié sur ais de bois, de veau estampé à froid, par une reliure du XIX ${ }^{\mathrm{e}}$ siècle en bon état ${ }^{8}$.

\section{Foliotation}

Le manuscrit compte 239 feuillets foliotés dès le XIV ${ }^{\mathrm{e}}$ siècle ${ }^{9}$, quoique cette foliotation ne recouvre pas tout le volume - elle concerne les feuillets 1 à 96 et 173 à 180 . $\mathrm{Au} \mathrm{XV}$ e siècle, une autre foliotation s'y est surimposée, conséquence probable de la confection d'une nouvelle reliure: on la trouve à partir du feuillet 97 jusqu'à la fin du volume, elle double celle du XIV ${ }^{\mathrm{e}}$ siècle, en la corrigeant, du feuillet 173 au folio $175^{10}$.

\section{- L'agencement des cahiers}

L'examen de la foliotation du manuscrit indique que l'ordre primitif des cahiers constituant le cartulaire a été modifié, vraisemblablement au XV siècle. Le volume comporte vingt-huit cahiers de parchemin: les vingt premiers cahiers correspondent au noyau primitif du cartulaire rédigé au XIII ${ }^{e}$ siècle.

Parmi ces vingt cahiers, les dix premiers appartiennent à la première campagne de copie, viennent alors deux cahiers prévus à cette même époque pour poursuivre la rédaction du manuscrit, ce qui fut fait. Dès cette époque, on avait prévu de laisser des feuillets vierges pour poursuivre, à l'avenir, la rédaction du cartulaire, considéré alors comme un instrument évolutif. Suivait encore, au XIII e siècle, un cahier supplémentaire prévu pour suivre les deux précédents, mais aujourd'hui rejeté plus loin dans le manuscrit. Enfin, les sept autres cahiers qui suivent correspondent à la campagne originelle de copie.

6. Cette description du cartulaire de Préaux reprend la substance des conclusions tirées dans le cadre de ma thèse d'École des chartes, Le cartulaire de Saint-Pierre de Préaux : étude et édition du manuscrit dans son état de 1227, Paris, 1999 (thèse dactylographiée, projet de publication en cours); voir aussi RouET, Dominique, «Le cartulaire de Saint-Pierre de Préaux : étude et édition du manuscrit dans son état de 1227 », Positions des thèses soutenues par les élèves de la promotion de 1999 pour obtenir le diplôme d'archiviste paléographe, Paris, 1999, p. 341-347.

7. $20,5 \mathrm{~cm}$ de largeur sur $28,5 \mathrm{~cm}$ de hauteur.

8. Cette reliure fut exécutée en 1859 par le relieur Guignard, comme l'indique une discrète dorure placée au bas du premier plat intérieur. Elle a remplacé celle qui, au XV siècle, était ornée de fermoirs d'argent à lacs de soie (voir thèse citée note 6, t. I, p. 122).

9. La graphie employée est très proche de celle d'une série de chartes copiées dans le cartulaire au XIV siècle.

10. On doit rapprocher l'écriture employée à celle présente dans la première partie du second cartulaire de Préaux datable de la fin de la première moitié du XV siècle et, de ce fait, on peut situer sans grand risque cette seconde campagne de foliotation autour de 1450 (BnF, nouv. acq. lat. 1929). 
Parmi les huit cahiers suivants, six ont été ajoutés au début du XIV ${ }^{\mathrm{e}}$ siècle ${ }^{11}$ et deux l'ont été au XV siècle.

\section{Les rubrications}

Le noyau originel du cartulaire se compose de chartes et de notices qui ont en commun d'être présentées de la même manière: elles ont été écrites par une seule main, sur une seule colonne qui occupe toute la largeur du feuillet ${ }^{12}$. Le copiste du cartulaire a peint certaines rubrications, mais c'est à un second moine que l'on doit la majeure partie d'entre elles. Ce dernier travailla après que le cartulariste eut terminé la copie des actes: il dut être actif durant la période 1229-1239. Sa graphie et ses habitudes d'écriture sont caractéristiques : peut-être d'origine anglaise, il substitue presque systématiquement aux $\mathrm{V}$ des $\mathrm{W}$, ce qui est particulièrement remarquable dans les noms de villages se terminant la plupart du temps par le suffixe -villa ${ }^{13}$.

Le cartulaire a été copié, on le verra, en 1227, mais diverses mains se sont ensuite relayées pour le compléter durant les XIII ${ }^{\mathrm{e}}$ et $\mathrm{XIV}^{\mathrm{e}}$ siècles. Le contenu du cartulaire tel qu'il se présentait en 1227 se présente de la façon suivante: il est clairement divisé en deux parties. La première comporte les transcriptions de chartes, bulles pontificales, actes épiscopaux, chartes royales, chartes des comtes de Meulan et de leur famille. La seconde partie du manuscrit est un enchaînement de notices relatant les acquisitions faites par les moines depuis la fondation de l'abbaye. Ces deux parties du cartulaire suivent deux logiques différentes quant à leur contenu et à la présentation des actes.

\section{Les conditions de la rédaction : le cartulaire dans son contexte historique}

L'entreprise de rédaction du premier cartulaire de Préaux a débuté sous l'abbatiat et, sans doute, sur l'ordre de l'abbé Bernard, appelé aussi Bernard de Combon par les auteurs de la Neustria pia et du Gallia christiana, bien que dans le cartulaire lui-même il ne soit jamais désigné par ce nom ${ }^{14}$. Le contexte de la rédaction du cartulaire correspond pour Saint-Pierre de Préaux à une phase de réorganisation du temporel après la période agitée du rattachement de la Normandie au domaine royal.

Le doute plane, en outre, sur les conditions dans lesquelles un des prédécesseurs de Bernard a dirigé l'abbaye. L'abbé Guillaume II (1200-1206) est mis en cause par

11. Leur rédaction et insertion dans le cartulaire à cet endroit doivent être datées du tout début du $\mathrm{XIV}^{\mathrm{e}}$ siècle : le moine qui les a transcrits a aussi copié à la fin du troisième cahier trois actes datés de 1302.

12. $21 \mathrm{~cm}$ environ de hauteur ; largeur: $14,5 \mathrm{~cm}$.

13. Par exemple le $\mathrm{n}^{\circ} \mathrm{A} 144$ de notre édition dont la rubrique est: «Ex dono Osberni de Magniwilla, terram duorum virorum unum in portaria super willam, alterum in Magna Willa».

14. DumOnstier, A., Neustria pia, p. 512; Gallia christiana, t. XI. col. 839. 
les auteurs du Gallia christiana. D'ailleurs, dès le début de l'abbatiat de Bernard, en 1221, les rôles de l'Échiquier prévoient un examen des chartes produites par l'abbaye, afin de déterminer celles qui l'ont été au détriment de la communauté, preuve d'une gestion hasardeuse du temporel sous le précédent abbatiat ${ }^{15}$.

L'abbé Bernard paraît, d'après les sources dont on dispose, s'être beaucoup investi en faveur de la gestion du temporel de l'abbaye: il effectua, en 1227, un voyage en Angleterre pour visiter les prieurés que l'abbaye y possédait, démarche qui n'a pas été réitérée systématiquement par ses successeurs, en dépit des injonctions faites en 1249 par l'archevêque de Rouen Eudes Rigaud en visite à Préaux ${ }^{16}$. L'abbé Bernard est aussi mentionné dans une rubrication du cartulaire pour s'être particulièrement investi dans la gestion des biens que le monastère possédait à Rouen ${ }^{17}$.

\section{Le copiste}

Le cartulaire de Préaux, tel qu'il se présentait dans son état originel, fut l'œuvre d'un seul copiste, même si celui-ci délégua une partie des rubrications à un autre moine. On laisse de côté ici les diverses mains qui ont continué d'étoffer le manuscrit au cours des XIII', XIV $^{\mathrm{e}}$ et XV $\mathrm{XV}^{\mathrm{e}}$ siècles.

Le moine copiste chargé de ce travail est un certain frère Guillaume, moine claustral de Saint-Pierre de Préaux, ainsi qu'il se qualifie lui-même: son nom ne nous serait jamais parvenu s'il ne l'avait inséré entre deux actes. Il mena à bien son œuvre à la fin de l'année 1227, voire au début de 1228. Sa première intervention dans le cartulaire est placée au folio $48 \mathrm{v}$ : introduite par un titre inséré après coup par le second rubricateur ${ }^{18}$, elle se place à la suite d'une confirmation du comte Roger de Warwick concernant les biens que l'abbaye possédait en Angleterre, à Warmington ${ }^{19}$. Cette mention est datée du 17 juin 1227, en l'absence de l'abbé Bernard dépêché en Angleterre pour visiter les prieurés que l'abbaye de Préaux y possédait.

La seconde intervention du copiste, datée du 9 août 1227 et située au folio $63 \mathrm{v}$, à la suite d'une donation de Geoffroi Ferrant ${ }^{20}$, annonce la fin du périple anglais de l'abbé Bernard et son retour à l'abbaye accompagné du moine de Préaux Adam de

15. Pâques 1221, échiquier de Caen : «Preceptum est quod omnes illi qui habent cartas de abbate et conventu de Pratellis cartas illas afferant ad instans scacarium de termino Sancti Michaelis instantis, ut per eas possit cognosci coram mandato domini regis que illarum facte sunt ad dampnum ecclesie et que non » (Delisle, Léopold., Recueil des jugements de l’Echiquier..., n 306, p. 77).

16. Registrum visitationum archiepiscopi Rothomagensis. Journal des visites pastorales d'Eudes Rigaud, archevêque de Rouen, 1248-1269, éd. Théodore BonNIN, Rouen, 1852, p. 295.

17. H 711 , fol. $80, \mathrm{n}^{\circ} 209 / \mathrm{n}^{\circ} \mathrm{B} 184$ : « Re vera, Bernardus, tunc temporis abbas de Pratellis, multum laboravit pro terra et domibus quas quondam tenuit magister Osmundus phisicus. Quas domos postea predictus $B$ [ernardus] donavit magistro Seobaldo pro suo servicio, ita scilicet quod predicte domus post mortem predicti Seobaldi revertentur cum omni sua melioratione ad monachos de Pratellis».

18. "Somnium magistri Willelmi scriptoris hujus libri».

19. H 711, fol. 48v, n ${ }^{\circ}$ 107. Warmington, Angleterre, co. Warwickshire. Voir ma thèse, t. I, p.127-128.

20. H 711, fol. $63 \mathrm{v}, \mathrm{n}^{\circ}$ 156. Dans le cartulaire nouv. acq. lat., 1929, cette intervention du copiste est glosée dans l'index des chartes par la mention: Ibi fit inventio de vasa in quo servatur S [anctissimum] sacramentum (fol. V). 
Cormeilles, ancien prieur de Toft Monks, prieuré que Préaux possédait en Angleterre, dans le Norfolk, d'où il rapporte une patène.

\section{Date de la copie}

De l'analyse des deux interventions directes du copiste où il rend, compte malgré lui, de l'avancée de son travail, on peut déduire la vitesse moyenne de copie du frère Guillaume : entre le 17 juin et le 9 août 1227, soit en 54 jours, 49 actes ou 30 pages ont été copiées. Cela paraît certes très peu, mais on doit, à la décharge du copiste, tenir compte du temps variable passé à trier et classer les chartes qu'il avait sous la main, des dimanches et des fêtes, jours pendant lesquels le copiste devait assurément suspendre son ouvrage, enfin, de la faible plage horaire que le moine pouvait consacrer à la copie.

Guillaume n'a pas copié d'acte postérieur au 11 juin $1227^{21}$ : on peut se demander pourquoi il s'est arrêté ici. En effet, on sait par ailleurs que le 9 août il était encore loin d'avoir terminé et qu'il n'y a pas de raison pour que la production de chartes de donation se soit arrêtée pendant l'été. En fait, il semble bien qu'il ait été occupé à copier la partie consacrée aux actes anciens : l'ordre des cahiers du manuscrit originel semble rendre compte du déroulement de la copie ; après avoir commencé par transcrire les actes les plus importants, Guillaume a dû recopier la partie consacrée aux notices plus anciennes.

On peut avoir une idée approximative de la durée du labeur de Guillaume, si l'on suppose ce travail comme ayant été continu: rien dans le manuscrit ne laisse croire l'inverse, bien au contraire, l'unité de la graphie incite à penser que la rédaction eut lieu d'un seul trait.

94 pages ont été copiées avant le 17 juin : il aurait donc fallu 169 jours environ à notre moine pour le faire, la copie a pu alors commencer au début de janvier 1227. De même, 101 pages ont été copiées après le 9 août 1227, ce qui lui ferait terminer son ouvrage vers le 5 janvier 1228. On peut alors considérer sans trop de risque que la première campagne de rédaction du cartulaire occupa globalement l'année 1227.

\section{Le contenu du cartulaire tel qu'il se présentait en 1227}

L'organisation interne du cartulaire se divise en deux parties (on laisse ici de côté les continuations des XIII- ${ }^{\mathrm{e}} \mathrm{XV}^{\mathrm{e}}$ siècles) : la première, compilée par Guillaume, est consacrée à des chartes datant pour la plupart de la seconde moitié du XII ${ }^{\mathrm{e}}$ siècle et du début du XIII ${ }^{e}$ siècle, jusqu'en 1227, en tout 202 actes. Le classement des chartes y est thématique, globalement rigoureux, mais souffre quelques exceptions : viennent en tête les bulles pontificales (Alexandre III, Lucius III, Célestin III, Innocent III

21. L'acte le plus récent copié dans ce cartulaire est daté de la veille de la Saint-Barnabé 1227, soit le 11 juin 1227 (H 711, fol. 68-v, n 171/nº B188 de notre édition). 
et Honorius III), les actes des évêques de Lisieux (Arnoul, Raoul de Varneville, Guillaume, Jourdain du Hommet, Guillaume II), d'Évreux et des archevêques de Rouen (Hugues d'Amiens, Rotrou de Warwick, Robert Poulain, Thibaud d'Amiens), entrecoupés par des règlements d'affaires obtenus devant des juges délégués pontificaux. Après les actes d'autorités ecclésiastiques sont transcrites les chartes royales de Henri II et de ses fils (Henri le jeune, Richard Cœur-de-lion), enfin les actes des comtes de Meulan: Galeran II et son fils Robert IV; suivent enfin les chartes de bienfaiteurs laïcs divers.

La seconde partie, également rédigée par Guillaume, commençant au feuillet 97, constitue la part la plus intéressante du cartulaire de Préaux: on y compte 200 actes, des notices pour la majorité d'entre eux, retraçant les acquisitions faites par les moines depuis la fondation de l'abbaye en 1034 jusqu'à l'abbatiat de Michel du Bec (1152-1167). Ces notices suivent un classement globalement géographique, qui met en évidence plusieurs dossiers concernant chacune un même lieu, à l'intérieur desquelles le classement est globalement chronologique; nombreuses cependant sont les exceptions, les notices inclassables, ou copiées plusieurs fois, ce qui trahit un remaniement en partie attribuable au cartulariste.

L'originalité de ce cartulaire réside dans cette seconde partie du manuscrit constituant un recueil de notices des $\mathrm{XI}^{e}$ et $\mathrm{XII}^{\mathrm{e}}$ siècles, vestige d'un ancien rouleau ${ }^{22}$, dont la teneur sans doute largement remaniée, n'est pas sans rappeler celle du rouleau de Saint-Évroult, conservé à la Bibliothèque nationale de France ${ }^{23}$. Le cartulaire de Préaux confectionné en 1227 réalise ainsi la synthèse d'un ancien cartulaire chronique du XII ${ }^{e}$ siècle, où la conception du cartulaire comme outil historiographique est manifeste, et de chartes nouvelles compilées au XIII siècle dans une optique plus gestionnaire.

\section{Exemples d'actes extraits du cartulaire}

Parmi les 403 actes que comprenait le cartulaire en 1227, près de 200 sont des notices datant des $\mathrm{XI}^{\mathrm{e}}$ et XII ${ }^{\mathrm{e}}$ siècles : rédigées sous un mode narratif, avec parfois des apartés dus au scripteur, elles s'enchaînent les unes aux autres comme le feraient les articles d'une chronique relatant l'évolution et l'histoire de la constitution du patrimoine de l'abbaye depuis sa fondation (voir A 59). L'ordre de classement n'est pas strictement chronologique ni même toujours cohérent, il trahit des réécritures, des reclassements effectués avant même le travail du cartulariste.

Les notices du recueil comportent rarement une date millésimée (celles-ci apparaissent au XII siècle, voir A31), au contraire, l'époque de l'action qu'elles rapportent est bien souvent située par l'évocation d'un événement (voir actes A59, A61), par la mention du duc régnant, ou de l'époque d'un abbé. Les listes de témoins, parfois

22. Deux notices du cartulaire citent explicitement l'existence du rouleau ( $\mathrm{n}^{\circ} \mathrm{A} 42$ et A44 de notre édition).

23. BnF, nouv. acq. lat. 2527. 
abondantes, et mettant souvent en scène des familiers de l'abbaye permettent également, par recoupement, de dater ces actes. Autre caractéristique, ces notices comportent des localisations bien souvent vagues et laconiques; elles mettent plutôt l'accent sur l'identité des donateurs, leur motivation, la traditio, les témoins de la cérémonie.

Les quatre notices ici éditées sont précédées de leur numérotation d'ordre dans la thèse citée plus haut ${ }^{24}$.

\section{Quelques notices extraites du cartulaire de Saint-Pierre de Préaux}

$\mathbf{N}^{\circ}$ A59

[...1066-1094]

Geoffroi [ [I"r], fils d'Osberne de Tourville, confirme avec l'accord de Roger de Beaumont et de ses fils Robert et Henri, les dons faits à l'abbaye Saint-Pierre de Préaux par son père Osberne, fils de Duveline: ce dernier avait donné la dîme et la mouture d'un vavasseur nommé Rainaud, du consentement de Roger de Beaumont et de ses fils.

$B$ : Cartulaire Arch. dép. Eure, $\mathrm{H} 711$, fol. 112v, n 336. En rubrique: «Ex dono Osberni de Turwilla decimam et molturam cujusdam wawassoris ». [Copie Delisle, BnF, nouv. acq. lat. 1025, fol. 142-143, n 336$]$.

C: Cartulaire du XVe siècle, BnF, nouv. acq. lat.1929, fol.73, nº 242.

Geoffroi I ${ }^{\text {er }}$ de Tourville, fils d'Osberne de Tourville est mort vers 1124 après avoir eu les deux yeux crevés sur l'ordre du roi Henri I ${ }^{\text {er }}$. Son père est attesté vers 1050 (DUMONSTIER, A., Neustria pia..., p. 522), mais est mort avant 1094, date du décès de Roger de Beaumont qui préside à la confirmation relatée dans cette notice; une autre version de cet acte est transcrite dans le cartulaire ( $\mathrm{n}^{\circ} \mathrm{A} 39$ de notre édition). Le souverain dont il est question dès le début de l'acte est Guillaume le Conquérant, d'après les notices qui précèdent celle-ci dans le cartulaire.

Eodem ${ }^{(a)}$ iterum principe regnante, Osbernus de Turvilla, filius Duveline, dedit Sancto Petro de Pratellis decimam et molturam cujus sui vavasoris, nomine Rainaldi, pro anima patris sui et matris sue, teste ${ }^{(b)}$ Heleboldo, fratre suo, et Roberto, dapifero, et Gisleberto filio Audomeri. Testes Sancti Petri : Gislebertus Chiderun; Robertus, portarius.

Mortuo vero predicto Osberno, Gaufridus, filius ejus, concessit et confirmavit donum patris sui signo crucis et posuit super altare per unum candelabrum, teste ${ }^{(c)}$ Walterio, granceario ; Moyse; Burnulfo, bubulco. Hec dona duo predicta, assistente et concedente Rogerio Belli Montis cum filiis suis, Roberto et Henrico, firmata sunt.

(a). eodem suivi de tempore biffé dans B. - (b). Sic B, C. - (c). Sic B, C.

24. Voir note $n^{\circ} 6$. 


\section{$\mathrm{N}^{\circ} \mathrm{A64}$}

1091

Geoffroi et Roger, fils de Gilbert, tous deux chevaliers, donnent à l'abbaye SaintPierre de Préaux une acre de terre sise en leur domaine de Campigny', pour l'âme de leur frère Robert Belet récemment tué à Évreux. Ils ajoutent sept autres acres que leur père avait depuis longtemps engagé auprès des moines contre cinquante sous de roumois qu'il avait reçus de son vivant.

$B$ : Cartulaire Arch. dép. Eure, $\mathrm{H} 711$, fol.113v, $\mathrm{n}^{\circ}$ 341. En rubrique: « Ex dono Gaufridi et Rogerii pro anima Roberti Beleit primum unum agrum postea VII, apud Campiniacum». [Copie Delisle, BnF, nouv. acq. lat. 1025, fol. 145-146-, $\left.\mathrm{n}^{\circ} 341\right]$.

C: Cartulaire du XVe siècle, BnF nouv. acq. lat.1929, fol.73v, nº 247.

Henri I ${ }^{e r}$ fut assiégé au Mont-Saint-Michel par ses frères Guillaume II, roi d'Angleterre, et Robert Courte-Heuse pendant tout le Carême 1091 (du 26 février au 13 avril 1091); la réconciliation entre les frères intervint avant l'été (DAVID, Charles Wendel, Robert Curthose Duke of Normandy, Cambridge, Harvard University Press, 1920, p. 62-64-65).

Anno quo Willelmus Rufus, rex Anglorum, et Robertus, comes Normannorum, obsederunt suum fratrem Henricum in Monte Sancti Michaelis, duo fratres, scilicet Gaufridus $^{(a)}$ et Rogerius, filii Gisleberti, venerunt in capitulum monachorum Pratellensium et pro anima Roberti Beleth, fratris eorum recenter interfecti apud Ebroicas, et pro animabus suorum parentum per unum cutellum portantes atque ponentes super altare sancti Petri, astante omni conventu et multis laicis, dederunt perpetualiter sancto Petro et monachis agrum terre quem in suo dominio habebant in Campiniaco et illos septem alios agros terre quos pater eorumdem militum abbati Pratellensi et monachis posuerat jamque diu in vadimonium tenuerant pro quinquaginta solidis romeisinorum. Hos autem denarios predictus Gislebertus ab abbate et a monachis receperat cum adviveret. Hujus donationis testes affuerunt ex parte ipsorum: Willelmus, nepos et armiger eorum ${ }^{(b)}$; Ricardus Wanescrot; ex parte abbatis: Willelmus Maledoctus; Radulfus Cocus; Hunfridus, hospitator; Gaufridus Polardus; Rogerius filius Christiani; Ascelinus; Postellus Parvulus.

(a). Gaufriduns B, corrigé dans C. - (b). La ponctuation du cartulaire, pour fiable qu'elle soit, invite à rapprocher nepos et armiger eorum de Guillaume plutôt que de Richard Wanescrot.

1. Campigny, Eure, cant. Pont-Audemer. 


\section{$\mathrm{N}^{\circ} \mathrm{A} 31$}

1120 (ou 1130 ?)

Osberne de Saint-Samson ${ }^{1}$ donne en aumône à l'abbaye Saint-Pierre de Préaux, avec l'accord de son épouse Havoise et en présence d'une foule de moines, clercs et laïcs, six acres de terre et le paysan y demeurant. Il fait ce don parce que Robert, moine de Préaux, a construit l'église paroissiale de Saint-Samson [-sur-Risle]. Il ajoute aussi une autre église, située non loin du village, [dédiée à saint Bérenger].

B: Cartulaire Arch. dép. Eure, H 711, fol. 106, n³12. En rubrique: «De Roca. Quomodo Sanson dedit nobis sex acras terre et unum rusticum supermanentem ». [Copie Delisle, BnF, nouv. acq. lat., 1025, fol. 127-128, n 312].

C: Cartulaire du XVe siècle, BnF, nouv. acq. lat., 1929, fol.68, nº 215. Dans la marge : «Brothonne » barré et corrigé en «La Roqueou etoit la chapelle de saint Berenger ».

Une inscription qui se trouvait dans l'église de Saint-Samson-sur-Risle (voir LE PrÉVOST, Auguste, Mémoires (...) pour servir à l'histoire du département de l'Eure, 1862-1869, t. III, p. 203), mentionnait la date de la dédicace de l'église qui eut lieu le 6 décembre 1129, célébrée par l'archevêque de Dol Baudri de Bourgueil; Saint-Samson faisait en effet partie de l'exemption de Dol. Quelques mois après, le 5 janvier 1130, Baudri mourut et fut inhumé dans l'abbatiale de Préaux.

On peut se demander pourquoi près de dix ans ont séparé l'achèvement de cette église, ici sous-entendu, et la cérémonie de dédicace. Faut-il soupçonner une erreur de transcription du copiste du cartulaire qui aurait oublié un X en recopiant la date? Le paysan donné ici en aumône est-il le même que celui évoqué dans la notice suivante, auquel cas, cette donation aurait effectivement eu lieu en 1130 ?

Anno ab Incarnatione Domini $\mathrm{M}^{\circ} \mathrm{C}^{\circ} \mathrm{XX}^{\circ}$, Osbernus Sancti Sansonis cum uxore sua Hatvidis venerunt Pratellum et coram magna multitudine monachorum et clericorum et laicorum dedit sancto Petro super altare in elemosina sex acras terre et unum rusticum desuper manentem. Ideo enim ita egit, quia Robertus, noster monachus, ecclesiam parrochie totam a fundamento construxit, et aliam ecclesiam, que ${ }^{(a)}$ longiuscule a villa aberat, similiter in elemosinam tribuit ${ }^{(b)}$. Testes ex parte monachorum $^{(c)}$ : Willelmus Albe Vie; Osbernus filius Hunfridi et Herluinus filius Radulfi Coci et Ricardus filius Hatvidis; Willelmus filius Caveller ${ }^{\text {(d) }}$; Tustinus Male doctus; ex parte Osberni : Fulco, presbiter; Radulfus Rufus; Alnaricus ${ }^{(e)}$, nepos Osberni ; Robertus Brito.

(crux) Signum Osberni de Sancto Sansone. (crux) Signum Amalrici ${ }^{(f)}$. (crux) Signum Hatvidis, uxoris ejus. Signum (crux) Radulfi, filii eorum. (crux) Signum Ricardi filii Herberti.

(a). qua corrigé en qui dans B; corrigé dans $C$ en que. - (b). Sancti Berengerii, ajout du XIVe siècle dans la marge de droite de B. - (c). monachus $B$, corrigé dans Cen monachorum. - (d). Sic B, Cavelier C. - (e). Sic B, C. — (f). Almarici C.

1. Saint-Samson-sur-Risle, Eure, cant. Quillebeuf-sur-Seine, com. Saint-Samsonde-La-Roque. 


\section{$\mathrm{N}^{\circ} \mathrm{A} 32$}

$[1130-1131 / 1144]$

Robert, prieur de l'abbaye Saint-Pierre de Préaux, reçoit Osberne [de Saint-Sanson] de la main de Robert le Breton: avec l'accord de son seigneur l'archevêque de Dol Geoffroi ${ }^{1}$, Osberne devient moine. Pendant sa maladie, cause de sa mort, sur les conseils de l'archevêque, Osberne avait donné à l'abbaye un hôte nommé Durand Malpuint et toute sa terre, dont les moines avaient été investis par son prévôt Rainfroi; Havoise, épouse d'Osberne, et Raoul son fils avaient donné leur accord.

$B$ : Cartulaire Arch. dép. Eure, H 711, fol. 106v, $\mathrm{n}^{\circ} 312$ bis. En rubrique: «Item de Roca. De Durando Malpuint». [Copie Delisle, BnF, nouv. acq. lat., 1025, fol. 128, $\left.\mathrm{n}^{\circ} 312\right]$.

$C$ : Cartulaire du XVe siècle, BnF, nouv. acq. lat., 1929, fol. 68, nº 216. Dans la marge: «Brothonne » barré et surmonté de «La Roque».

La datation de cette notice est déterminée par l'épiscopat de Geoffroi, l'archevêque de Dol entre 1130 et 1144. Il succéda à Baudri de Bourgueil qui se fit enterrer dans l'abbatiale de Saint-Pierre de Préaux, devant le crucifix du choeur (Cf. A. N, M725, fol.17 et Le PrÉvost, Auguste, Mémoires (...) pour servir à l'histoire du département de l'Eure, 1862-1869, t. III, p. 203).

L'entrée d'Osberne de Saint-Samson dans la communauté monastique de Préaux eut lieu avant 1135: entre 1135 et 1150, son fils Raoul de Saint-Samson apparaît comme ayant déjà hérité des terres de son père, donnant son accord à la concession de terres sises à Saint-Samson par Richard Broc en faveur des lépreux de PontAudemer (MESMIN, Simone, «Du comte à la Commune: La léproserie de SaintGilles de Pont-Audemer", Annales de Normandie, mai 1987, p. 257-258).

Raoul le Roux, témoin de cette charte et de la précédente, est neveu de l'archevêque de Dol Baudri de Bourgueil et frère de l'archevêque Geoffroi. Il atteste en 1141 un acte de son frère en faveur de Saint-Sauveur de La Vieuville (MORICE, Dom Hyacinthe, Mémoires pour servir de preuves à l'histoire (...) de Bretagne..., t. I, Paris, 1742, coll. 582).

Supradictus Osbernus, infirmitate cogente qua et mortuus est et Goisfredo, archiepiscopo Dolensi, domino ejus dante consilium, donavit sancto Petro et monachis ejus unum hospitem et totam terram suam, nomine Durandum Malpuint, jure elemosine, tenente unam virgam ex una parte et Hatvide, ejus uxore, ex altera parte et concedente et donante pariter istud et filio eorum Rodulfo. In eo die fecerunt nos saisire de hoc homine per Rainfredum suum prepositum.

Mox denique, jussu archiepiscopi, Robertus Brito Roberto priori liberavit Osbernum per manum, ut faceret monachum. Hoc factum est concessu et admonitione jam dicti archiepiscopi G [oisfredi]. Testes ex parte Osberni : Amlricus ${ }^{(a)}$; Ricardus filius Herberti; Fulco, presbiter; Robertus Hait; Hatvidis; Agnes, filia ejus. Testes Sancti Petri : Radulfus Rufus, nepos Baldrici episcopi ; Ricardus Nanus; Herluinus, camerarius; Goisfredus de Mara cum aliis multis.

Signum (crux) archiepiscopi. (crux) Signum Osberni. Signum (crux) Rodulfi, filii ejus. (crux) Signum Amalrici ${ }^{(\mathrm{b})}$. Signum (crux) Hatvidis.

(a). Sic B, C. - (b). Almrici C.

1. Geoffroi, archevêque de Dol, 1130 - 1144. 